\title{
Small oscillations of non-dissipative Lagrangian systems
}

Cite as: J. Math. Phys. 60, 042902 (2019); https://doi.org/10.1063/1.5049438

Submitted: 21 July 2018 . Accepted: 03 April 2019 . Published Online: 22 April 2019

Enrico Massa (D), and Stefano Vignolo (D)

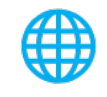

Where in the world is AIP Publishing? Find out where we are exhibiting next 


\title{
Small oscillations of non-dissipative Lagrangian systems
}

\author{
Cite as: J. Math. Phys. 60, 042902 (2019); doi: 10.1063/1.5049438 \\ Submitted: 21 July 2018 • Accepted: 3 April 2019 • \\ Published Online: 22 April 2019
}

\section{Enrico Massa ${ }^{a)}$ (D) and Stefano Vignolo}

\section{AFFILIATIONS}

DIME, Sez. Metodi e Modelli Matematici, Università di Genova, Via All'Opera Pia 15, 16145 Genova, Italy

a) Electronic mail: massa@dima.unige.it
b) Electronic mail: vignolo@dime.unige.it

\section{ABSTRACT}

The small oscillations of an arbitrary scleronomous system subject to time-independent nondissipative forces are discussed. The linearized equations of motion are solved by quadratures. As in the conservative case, the general integral is shown to consist of a superposition of harmonic oscillations. A complexification of the resolving algorithm is presented.

Published under license by AIP Publishing. https://doi.org/10.1063/1.5049438

\section{INTRODUCTION}

The concept of equilibrium stability and the associated theory of small oscillations are standard topics in classical analytical mechanics.

Surprisingly enough, in the literature, with a few notable exceptions, the second issue is dealt with under hypotheses definitely more restrictive than those adopted in the study of the first one: typically, while a sufficient condition for stability is established for arbitrary, time independent Lagrangians of the form

$$
L=\frac{1}{2} a_{i j} \dot{q}^{i} \dot{q}^{j}+b_{i} \dot{q}^{i}+c:=L_{2}+L_{1}+L_{0}
$$

small oscillations are usually discussed under the simplifying assumption $L_{1}=0$-more specifically, assuming constraints at rest in an inertial frame of reference and conservative forces. ${ }^{1-5}$ Possible extensions to nonconservative systems are sometimes considered, ${ }^{6-9}$ mainly in connection with the presence of dissipative or gyroscopic effects.

In this paper, we propose an approach to the study of small oscillations for scleronomous systems obeying the evolution equations

$$
\frac{d}{d t} \frac{\partial L}{\partial \dot{q}^{k}}-\frac{\partial L}{\partial q^{k}}=Q_{k}
$$

with Lagrangian $L\left(q^{h}, \dot{q}^{h}\right)$ of the general form (1) and generalized forces $Q_{k}\left(q^{h}, \dot{q}^{h}\right)$ fulfilling the nondissipativity condition $Q_{k} \dot{q}^{k}=0 .{ }^{11}$

The resulting linearized equations of motion, viewed as first order differential equations in $\mathbb{R}^{2 n}$, are shown to be solvable by quadratures. As expected, the general solution is a linear superposition of harmonics, determined by the spectral structure of a symmetric, negative definite matrix, expressing the square of the evolution operator.

An alternative characterization of the harmonics based on a $n$-dimensional complex formalism is finally worked out. The relation of the latter with the $2 n$-dimensional real approach is discussed.

\section{SMALL OSCILLATIONS}

Under the assumptions stated in the Introduction, every strict local maximum $q^{*}=\left(q^{* 1}, \ldots, q^{* n}\right)$ of the function $L_{0}$ is readily seen to represent a stable equilibrium configuration for the given system.

In fact, in view of the condition $Q_{r} \dot{q}^{r}=0$ and of the consequent relation 


$$
0=\frac{\partial}{\partial \dot{q}^{k}}\left(Q_{r} \dot{q}^{r}\right)=Q_{k}+\frac{\partial Q_{r}}{\partial \dot{q}^{k}} \dot{q}^{r}
$$

the generalized forces do not play any role in the study of the equilibrium configurations, as well as in the applicability of Dirichlet's stability theorem.

The second-order approximation of the Lagrangian in a neighborhood of the kinetic state $\left(q^{*}, 0\right)$ reads

$$
\tilde{L}=\frac{1}{2} A_{k r} \dot{\eta}^{k} \dot{\eta}^{r}+\left[b_{r}\left(q^{*}\right)+\left(\frac{\partial b_{r}}{\partial q^{k}}\right)_{q^{*}} \eta^{k}\right] \dot{\eta}^{r}-\frac{1}{2} C_{k r} \eta^{k} \eta^{r}
$$

with $A_{k r}=a_{k r}\left(q^{*}\right), C_{k r}=-\left(\frac{\partial^{2} L_{0}}{\partial q^{k} \partial q^{r}}\right)_{q^{*}}, \eta^{k}=q^{k}-q^{* k}$

From the latter, we get the expressions

$$
\frac{d}{d t} \frac{\partial \tilde{L}}{\partial \dot{\eta}^{k}}-\frac{\partial \tilde{L}}{\partial \eta^{k}}=A_{k r} \ddot{\eta}^{r}+\left[\left(\frac{\partial b_{k}}{\partial q^{r}}\right)_{q^{*}}-\left(\frac{\partial b_{r}}{\partial q^{k}}\right)_{q^{*}}\right] \dot{\eta}^{r}+C_{k r} \eta^{r} .
$$

In a similar way, on account of Eq. (3), the first order approximation of the generalized forces in a neighborhood of $\left(q^{*}, 0\right)$ takes the form

$$
\tilde{Q}_{k}=\left(\frac{\partial Q_{k}}{\partial \dot{q}^{r}}\right)_{\left(q^{*}, 0\right)} \dot{\eta}^{r}=-\left(\frac{\partial Q_{r}}{\partial \dot{q}^{k}}\right)_{\left(q^{*}, 0\right)} \dot{\eta}^{r}
$$

Collecting all results and setting

the linearized equations of motion read

$$
B_{k r}:=\left(\frac{\partial b_{k}}{\partial q^{r}}\right)_{q^{*}}-\left(\frac{\partial b_{r}}{\partial q^{k}}\right)_{q^{*}}-\left(\frac{\partial Q_{k}}{\partial \dot{q}^{r}}\right)_{\left(q^{*}, 0\right)},
$$

or, synthetically,

$$
A_{k r} \ddot{\eta}^{r}+B_{k r} \dot{\eta}^{r}+C_{k r} \eta^{r}=0
$$

$$
A \underset{\sim}{\ddot{\eta}}+B \underset{\sim}{\dot{\eta}}+C \underset{\sim}{\eta}=0
$$

where $A$ and $C$ are symmetric, positive definite matrices, $B$ is an antisymmetric one, and $\underset{\sim}{\eta}$ is the column vector $\left(\begin{array}{c}\eta^{1} \\ \vdots \\ \eta^{n}\end{array}\right)$.

Viewed as a system of first order ordinary differential equations (ODE) in the velocity space, Eq. (4) may be written in the normal form

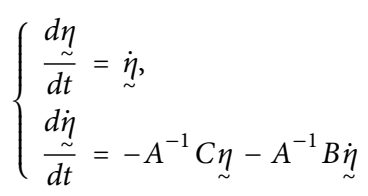

or in matrix notation

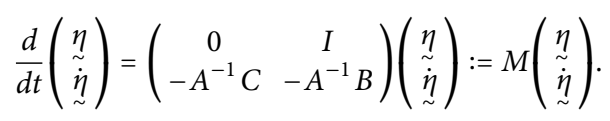

The nonsingular endomorphism $M: \mathbb{R}^{2 n} \rightarrow \mathbb{R}^{2 n}$ described by the matrix

$$
M=\left(\begin{array}{cc}
0 & I \\
-A^{-1} C & -A^{-1} B
\end{array}\right)
$$

will be called the linearized evolution operator.

In addition to $M$, another significant operator is the bilinear functional $\mathbb{R}^{2 n} \times \mathbb{R}^{2 n} \rightarrow \mathbb{R}$ sending each pair of vectors $\underset{\sim}{u}, \underset{\sim}{v}$ into the scalar ${ }^{t} \underset{\sim}{u} \underset{\sim}{v}, K$ denoting the nonsingular, antisymmetric matrix

$$
K:=\left(\begin{array}{cc}
B & A \\
-A & 0
\end{array}\right)
$$


For clarity, a few comments are in order:

- linear endomorphisms and bilinear functionals are of course different mathematical objects, with different composition rules: for example, in the present context, formally legitimate expressions like ${ }_{\sim}^{t} u M_{\sim} v$ or $K^{2}$ have no invariant geometrical meaning, while ${ }^{t} u K M \underset{\sim}{v}$ or $M^{2}$ are perfectly significant;

- every symmetric positive definite bilinear functional may be used to define a scalar product ( , ) over $\mathbb{R}^{2 n}$;

- a linear endomorphism $\psi: \mathbb{R}^{2 n} \rightarrow \mathbb{R}^{2 n}$ is symmetric (antisymmetric) with respect to a given scalar product if and only if the expression $(\underset{\sim}{u}, \psi \underset{\sim}{v})$ is symmetric (respectively, antisymmetric) in the arguments $\underset{\sim}{u} \underset{\sim}{v}$. In particular, if $\psi$ is symmetric, the space $\mathbb{R}^{2 n}$ admits an orthonormal basis formed by eigenvectors of $\psi$.

Straightforward consequences of the previous remarks are the assertions:

- the bilinear functional associated with the matrix $K M^{-1}$ is symmetric and positive definite. The conclusion follows at once from the identities

$$
\begin{gathered}
{ }^{t} K M=\left(\begin{array}{cc}
-B & -A \\
A & 0
\end{array}\right)\left(\begin{array}{cc}
0 & I \\
-A^{-1} C & -A^{-1} B
\end{array}\right)=\left(\begin{array}{ll}
C & 0 \\
0 & A
\end{array}\right), \\
K M^{-1}=K\left({ }^{t} K M\right)^{-1}{ }^{t} K=K\left(\begin{array}{cc}
C^{-1} & 0 \\
0 & A^{-1}
\end{array}\right){ }^{t} K,
\end{gathered}
$$

showing that $K \cdot M^{-1}$ is congruent to a symmetric, positive definite matrix;

- the operator $M$ is antisymmetric with respect to the scalar product defined by the prescription

$$
(\underset{\sim}{u}, \underset{\sim}{v}):={ }_{\sim}^{t} \underset{\sim}{u} K M^{-1} \underset{\sim}{v}
$$

Indeed, from Eq. (7) and the antisymmetry of $K$, it directly follows $(\underset{\sim}{u}, M \underset{\sim}{v})={ }^{t} \underset{\sim}{u} K \underset{\sim}{v}=-(\underset{\sim}{v}, M \underset{\sim}{u})$;

- the operator $M^{2}$ is symmetric and negative definite with respect to the scalar product (7): the antisymmetry and nonsingularity of $M$ entail in fact the relations

$$
\begin{aligned}
& \left(\underset{\sim}{u}, M^{2} \underset{\sim}{v}\right)=-(M \underset{\sim}{u}, M \underset{\sim}{v})=\left(M^{2} \underset{\sim}{u}, \underset{\sim}{v}\right), \\
& \left(\underset{\sim}{u}, M^{2} \underset{\sim}{u}\right)=-(M \underset{\sim}{u}, M \underset{\sim}{u})<0 \quad \forall \underset{\sim}{u} \in \mathbb{R}^{2 n}, \underset{\sim}{u} \neq \underset{\sim}{0} .
\end{aligned}
$$

After these preliminaries, we now state the following. relations

Theorem 1. The vector space $\mathbb{R}^{2 n}$ admits a basis $\underset{\sim}{\underset{\sim}{u_{k}}, \underset{\sim}{v}}, k=1 \ldots n$, orthonormal with respect to the scalar product (7) and satisfying the with $\omega_{1}, \ldots, \omega_{n}$ positive, not necessarily distinct real numbers.

$$
M \underset{\sim}{u_{k}}=\omega_{k}{\underset{\sim}{v}}_{k}, \quad M \underset{\sim}{v_{k}}=-\omega_{k} \underset{\sim}{u_{k}}
$$

Proof. Let $-\lambda_{\alpha}^{2}, \lambda_{\alpha} \in \mathbb{R}_{+}, \alpha=1, \ldots, r$, denote the distinct eigenvalues of $M^{2}$ and $\mathcal{S}_{\alpha} \subset \mathbb{R}^{2 n}$ denote the corresponding eigenspaces.

To each $\underset{\sim}{u} \in \mathcal{S}_{\alpha}$, we associate a "partner vector" $\underset{\sim}{v}:=\lambda_{\alpha}^{-1} M \underset{\sim}{u}$. The pair $\underset{\sim}{u}, \underset{\sim}{v}$ then satisfies the relations

$$
\begin{aligned}
& M \underset{\sim}{u}=\lambda_{\alpha} \underset{\sim}{v}, M \underset{\sim}{v}=\lambda_{\alpha}^{-1} M^{2} \underset{\sim}{u}=-\lambda_{\alpha} \underset{\sim}{u}, \\
& M^{2} \underset{\sim}{v}=-\lambda_{\alpha} M \underset{\sim}{u}=-\lambda_{\alpha}^{2} \underset{\sim}{v}, \\
& (\underset{\sim}{u} \underset{\sim}{v})=\lambda_{\alpha}^{-1}(\underset{\sim}{u}, M \underset{\sim}{u})=0, \\
& (\underset{\sim}{v} \underset{\sim}{v})=\lambda_{\alpha}^{-2}(M \underset{\sim}{u}, M \underset{\sim}{u})=-\lambda_{\alpha}^{-2}\left(\underset{\sim}{u}, M^{2} \underset{\sim}{u}\right)=(\underset{\sim}{u} \underset{\sim}{u}) .
\end{aligned}
$$

These show that, like $\underset{\sim}{u}$, the vector $v$ belongs to the eigenspace $\mathcal{S}_{\alpha}$ and that the vectors $\underset{\sim}{u}, \underset{\sim}{v}$ are mutually orthogonal, have the same norm, and fulfil an equation of the form (8) with $\omega_{k}$ replaced by $\lambda_{\alpha} \cdot{ }^{12}$ Moreover, setting $V:=\operatorname{Span}\{\underset{\sim}{u}, \underset{\sim}{v}\}$, the relations

$$
\begin{aligned}
& (M \underset{\sim}{w}, \underset{\sim}{u})=-(\underset{\sim}{w}, M \underset{\sim}{u})=-\lambda_{\alpha}(\underset{\sim}{w}, \underset{\sim}{v}), \\
& (M \underset{\sim}{w}, \underset{\sim}{v})=-(\underset{\sim}{w}, M \underset{\sim}{v})=\lambda_{\alpha}(\underset{\sim}{w}, \underset{\sim}{u})
\end{aligned}
$$

show that the operator $M$ maps the subspace $V^{\perp}$ onto itself, thereby inducing a nonsingular antisymmetric endomorphism $M_{\mid V^{\perp}}: V^{\perp} \rightarrow V^{\perp}$, whose square is clearly identical to the restriction $\left(M^{2}\right)_{\mid V^{\perp}}$.

The rest of the proof proceeds by induction: for $n=1$ (namely, in $\mathbb{R}^{2}$ ), choosing $\underset{\sim}{u}$ of unit norm, the ansatz $\underset{\sim}{\mathcal{u}}{ }_{1}=\underset{\sim}{\mathcal{u}} \underset{\sim}{v} v_{1}=\underset{\sim}{v}, \omega_{1}=\lambda$ establishes the thesis. 
In a similar way, for $n>1$, we arbitrarily select an eigenspace $\mathcal{S}_{\alpha}$ of $M^{2}$, a unit vector $\underset{\sim}{u_{n}} \in \mathcal{S}_{\alpha}$, and denote by $\underset{\sim}{v_{n}}=\lambda_{\alpha}^{-1} M \underset{\sim}{u_{n}}$ the associated partner vector. $V_{n}^{\perp}$.

The thesis then follows setting $V_{n}=\operatorname{Span}\left\{\underset{\sim}{u_{n}}, \underset{\sim}{v}\right\}, \omega_{n}=\lambda_{\alpha}$ and applying the inductive hypothesis to the (2n-2)-dimensional subspace

Introducing the notation

$$
\underset{\sim}{\mathcal{u}_{k}}=\left(\begin{array}{c}
\underset{\sim}{h_{k}} \\
\underset{\sim}{l_{k}}
\end{array}\right), \underset{\sim}{v_{k}}=\left(\begin{array}{c}
\underset{\sim}{r_{k}} \\
\underset{\sim}{s} k
\end{array}\right)
$$

with $\underset{\sim}{h_{k}}, l_{\sim}, r_{\sim}, s_{\sim}$ column vectors in $\in \mathbb{R}^{n}$, Eqs. (5) and (8) imply the equalities

$$
\begin{aligned}
& \left(\begin{array}{cc}
0 & I \\
-A^{-1} C & -A^{-1} B
\end{array}\right)\left(\begin{array}{c}
\underset{\sim}{h_{k}} \\
\underset{\sim}{l_{k}}
\end{array}\right)=\omega_{k}\left(\begin{array}{c}
\underset{\sim}{r_{k}} \\
\underset{\sim}{s} k
\end{array}\right), \\
& \left(\begin{array}{cc}
0 & I \\
-A^{-1} C & -A^{-1} B
\end{array}\right)\left(\begin{array}{c}
\underset{\sim}{r_{k}} \\
\underset{\sim}{s} k
\end{array}\right)=-\omega_{k}\left(\begin{array}{c}
\underset{\sim}{h_{k}} \\
\underset{\sim}{l_{k}}
\end{array}\right)
\end{aligned}
$$

summarized into the pair of expressions

completed by the complex relation

$$
\stackrel{\sim}{\sim}_{k}=\omega_{k} r_{\sim}, \quad{\underset{\sim}{s}}_{k}=-\omega_{k} \underset{\sim}{h} h_{k}
$$

$$
\left(C-i \omega_{k} B-\omega_{k}^{2} A\right)\left(\underset{\sim}{h}{ }_{k}+\underset{\sim}{\underset{\sim}{r}} k\right)=0 .
$$

Referring $\mathbb{R}^{2 n}$ to the basis $\underset{\sim}{u_{k}}, \underset{\sim}{v}$ indicated in Theorem 1 and putting

$$
\left(\begin{array}{c}
\underset{\sim}{\sim} \\
\underset{\sim}{\dot{\eta}}
\end{array}\right)=\chi_{k}(t) \underset{\sim}{\mathcal{u}_{k}}+\psi_{k}(t) \underset{\sim}{v_{k}}
$$

we rewrite the evolution equation $\left(4^{\prime}\right)$ in the form

$$
\dot{\chi}_{k}(t) \underset{\sim}{u_{k}}+\dot{\psi}_{k}(t) \underset{\sim}{v_{k}}=M\left[\chi_{k}(t) \underset{\sim}{u_{k}}+\psi_{k}(t) \underset{\sim}{v_{k}}\right]=\omega_{k}\left[\chi_{k}(t) \underset{\sim}{v_{k}}-\psi_{k}(t) \underset{\sim}{u_{k}}\right],
$$

mathematically equivalent to the system

The latter admits the general integral

$$
\left\{\begin{array}{l}
\dot{\chi}_{k}=-\omega_{k} \psi_{k} \\
\dot{\psi}_{k}=\omega_{k} \chi_{k}
\end{array} \quad \text { (not summed over } k\right)
$$

$$
\chi_{k}=a_{k} \cos \left(\omega_{k} t+\varphi_{k}\right), \quad \psi_{k}=a_{k} \sin \left(\omega_{k} t+\varphi_{k}\right)
$$

with $a_{k}, \varphi_{k}$ arbitrary constants. The solution of Eq. $\left(4^{\prime}\right)$ is therefore

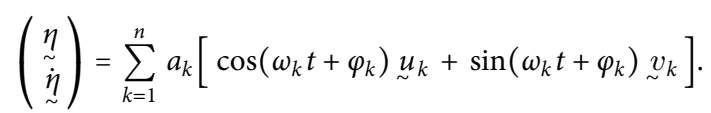

Recalling Eq. (9) we conclude that the general integral of the linearized equations of motion (4) reads

$$
\underset{\sim}{\eta}=\sum_{k=1}^{n} a_{k}\left[\cos \left(\omega_{k} t+\varphi_{k}\right) \underset{\sim}{h_{k}}+\sin \left(\omega_{k} t+\varphi_{k}\right) \underset{\sim}{\underset{\sim}{r}}\right],
$$

while the expression for $\dot{\eta}$ agrees with the one obtained evaluating the time derivative of the right-hand side of Eq. (12) and taking the identifications (10) into account.

As expected, the motion of the system consists of a linear superposition $\underset{\sim}{\eta}=\sum_{k} a_{k} \underset{\sim}{v_{k}}$ of harmonic oscillations, henceforth called the normal harmonics.

The determination of the general integral (12) can be simplified, replacing the $2 n$-dimensional real formalism with a $n$-dimensional complex one. 
To this end, resuming the notations adopted in the Proof of Theorem 1 , we indicate by $\mathcal{S}_{\alpha}, \alpha=1, \ldots, r$, the eigenspaces of the operator $M^{2}$, by $-\lambda_{\alpha}^{2}\left(\lambda_{\alpha}>0\right)$ the corresponding eigenvalues, and by $2 n_{\alpha}=\operatorname{dim}\left(\mathcal{S}_{\alpha}\right)$ the respective multiplicities. Also, for each $\alpha$, we denote by $\mathcal{N}_{\alpha}$ the kernel of the endomorphism $\left(C-i \lambda_{\alpha} B-\lambda_{\alpha}^{2} A\right): \mathbb{C}^{n} \rightarrow \mathbb{C}^{n}$.

We then have the following.

Theorem 2. The space $\mathbb{C}^{n}$ splits into the direct sum

$$
\mathbb{C}^{n}=\oplus_{\alpha=1}^{r} \mathcal{N}_{\alpha}
$$

with $\operatorname{dim}\left(\mathcal{N}_{\alpha}\right)=n_{\alpha}$.

Proof. To start with, we establish the intersection property $\mathcal{N}_{\alpha} \cap \mathcal{N}_{\beta}=\{0\} \forall \alpha \neq \beta$. To this end, we observe that, for any $z \in \mathcal{N}_{\alpha} \cap \mathcal{N}_{\beta}$, the simultaneous validity of the conditions

$$
\begin{aligned}
& \left(C-i \lambda_{\alpha} B-\lambda_{\alpha}^{2} A\right) \underset{\sim}{z}=0, \\
& \left(C-i \lambda_{\beta} B-\lambda_{\beta}^{2} A\right) \underset{\sim}{z}=0
\end{aligned}
$$

implies the equality

$$
\left[i\left(\lambda_{\alpha}-\lambda_{\beta}\right) B+\left(\lambda_{\alpha}^{2}-\lambda_{\beta}^{2}\right) A\right] \underset{\sim}{z}=\left(\lambda_{\alpha}-\lambda_{\beta}\right)\left[i B+\left(\lambda_{\alpha}+\lambda_{\beta}\right) A\right] \underset{\sim}{z}=0,
$$

whence dividing by $\lambda_{\alpha}-\lambda_{\beta}$ and substituting into Eq. (14a)

$$
\left[C+\lambda_{\alpha}\left(\lambda_{\alpha}+\lambda_{\beta}\right) A-\lambda_{\alpha}^{2} A\right]_{\sim}^{z}=\left(C+\lambda_{\alpha} \lambda_{\beta} A\right) \underset{\sim}{z}=0 .
$$

At the same time, the positiveness of $\lambda_{\alpha}, \lambda_{\beta}$, together with the positive definiteness of $A$ and $C$, entails the nonsingularity of the matrix $C+\lambda_{\alpha} \lambda_{\beta} A$. Therefore, $\underset{\sim}{z} \in \mathcal{N}_{\alpha} \cap \mathcal{N}_{\beta}$ if and only if $\underset{\sim}{z}=0$.

Let us now evaluate the dimension of each $\mathcal{N}_{\alpha}$. To this end, referred $\mathbb{R}^{2 n}$ to an orthonormal basis satisfying Eq. (8) we collect the basis vectors $\left\{\underset{\sim}{u_{k}}, v_{\sim}\right\}$ into $r$ distinct subfamilies $\left\{\underset{\sim}{u_{i_{\alpha}}^{(\alpha)}}, \underset{\sim}{v_{i_{\alpha}}^{(\alpha)}}, i_{\alpha}=1, \ldots, n_{\alpha}\right\}$, each one spanning a corresponding eigenspace $\mathcal{S}_{\alpha}$.

Restoring the notation (9), to each pair of "partner vectors" $\underset{\sim}{u} i_{\alpha}^{(\alpha)}, \underset{\sim}{v_{i_{\alpha}}^{(\alpha)}}$, we associate the complex vector $\underset{\sim}{i_{\alpha}}(\alpha)=\underset{\sim}{h_{i}}(\alpha)+\underset{\sim}{(\alpha)} \dot{\sim}_{i_{\alpha}}^{(\alpha)} \in \mathbb{C}^{n}$. In view of Eq. (11), $z_{i_{\alpha}}^{(\alpha)}$ belongs to the kernel $\mathcal{N}_{\alpha}$. Moreover, the vectors $\left\{z_{i_{\alpha}}^{(\alpha)}, i_{\alpha}=1, \ldots, n_{\alpha}\right\}$ are linearly independent: by Eq. (10), adapting the notation $\left(i_{\alpha}\right.$ in place of $k, \lambda_{\alpha}$ in place of $\left.\omega_{k}\right)$, we have in fact the identifications

$$
\underset{\sim}{u}(\alpha)+i \underset{\sim}{i i_{\alpha}}(\alpha)=\left(\begin{array}{c}
{\underset{\sim}{i_{\alpha}}}_{i_{\alpha}}^{(\alpha)} \\
-i \lambda_{\alpha} z_{i_{\alpha}}^{(\alpha)}
\end{array}\right) .
$$

In view of these, every relation of the form $\sum_{i_{\alpha}}\left(a_{i_{\alpha}}+i b_{i_{\alpha}}\right) z_{i_{\alpha}}^{(\alpha)}=0$ implies the validity of the equations

$$
\sum_{i_{\alpha}}\left(a_{i_{\alpha}} \underset{\sim}{\sim} i_{i_{\alpha}}^{(\alpha)}-b_{i_{\alpha}} \underset{\sim}{\sim} i_{i_{\alpha}}^{(\alpha)}\right)=\sum_{i_{\alpha}}\left(a_{i_{\alpha}} \underset{\sim}{\sim} i_{i_{\alpha}}^{(\alpha)}+b_{i_{\alpha}} \underset{\sim}{u_{i_{\alpha}}^{(\alpha)}}\right)=0
$$

which, on account of the linear independence of the vectors $\underset{\sim}{u_{i_{\alpha}}}(\alpha) \underset{\sim}{v_{i_{\alpha}}}(\alpha)$, ensure the vanishing of all coefficients $a_{i_{\alpha}}, b_{i_{\alpha}}$.

The dimension of $\mathcal{N}_{\alpha}$ is therefore not less than $n_{\alpha}$. Being $\sum_{\alpha} n_{\alpha}=n$, this fact, together with the intersection property, establishes the thesis.

According to Theorem 2, the space $\mathbb{C}^{n}$ admits at least one basis $\left\{z_{1}, \ldots, z_{n}\right\}$ whose elements satisfy equations of the form

$$
\left(C-i \omega_{k} B-\omega_{k}^{2} A\right) z_{k}=0
$$

$\omega_{k}$ being $n$ (not necessarily distinct) positive roots of the equation $\operatorname{det}\left(C-i \omega B-\omega^{2} A\right)=0$.

Equation (15) implies the differential relation

$$
\left(A \frac{d^{2}}{d t^{2}}+B \frac{d}{d t}+C\right)\left(e^{-i \omega_{k} t} z_{k}\right)=0
$$

as well as the complex conjugate one 


$$
\left(A \frac{d^{2}}{d t^{2}}+B \frac{d}{d t}+C\right)\left(e^{i \omega_{k} t} \bar{z}_{k}\right)=0 .
$$
integral

In the space $\mathbb{C}^{n}$, the second order differential equation $A \underset{\sim}{\ddot{z}}+B \underset{\sim}{\dot{z}}+C_{\sim} z=0$ for the unknown $\underset{\sim}{z}=\underset{\sim}{z}(t)$ admits therefore the general

$$
\underset{\sim}{z}=\sum_{k=1}^{n}\left(\gamma_{k} e^{-i \omega_{k} t} z_{k}+\delta_{k} e^{i \omega_{k} t} \bar{z}_{k}\right)
$$

$\gamma_{k}, \delta_{k}$ being arbitrary complex constants.

The right-hand side of (16) is real if and only if $\delta_{k}=\bar{\gamma}_{k}$. Setting $\gamma_{k}=a_{k} e^{-i \varphi_{k}}$ and restoring the notation $\underset{\sim}{\underset{\sim}{z}}=\underset{\sim}{h}+\underset{\sim}{i} r_{k}$, we obtain in this way the general integral of Eq. (4) in the form

$$
\underset{\sim}{\eta}(t)=\operatorname{Re} \sum_{k=1}^{n}\left[a_{k} e^{-i\left(\omega_{k} t+\varphi_{k}\right)}\left(\underset{\sim}{h_{k}}+\underset{\sim}{i} r_{k}\right)\right]
$$

clearly identical to Eq. (12).

Although not directly relevant to the implementation of the algorithm, it may be noticed that, unlike what happens in $\mathbb{R}^{2 n}$, the characterization of the bases $\left\{z_{1}, \ldots, z_{n}\right\}$ of $\mathbb{C}^{n}$ fulfilling the requirements (15) does not involve any concept of orthonormality.

This lack of symmetry between the real and the complex formalism may be disposed of by endowing each subspace $\mathcal{N}_{\alpha} \subset \mathbb{C}^{n}$ with a sesquilinear scalar product, based on the prescription

$$
(\underset{\sim}{z} \underset{\sim}{w})={ }^{t} \bar{\sim}\left(A+\frac{i B}{2 \lambda_{\alpha}^{2}}\right) \underset{\sim}{w}
$$

By the very definition of $\mathcal{N}_{\alpha}$, Eq. (17) entails the relation

$$
{ }^{t} \bar{\sim}\left(A+\frac{i B}{2 \lambda_{\alpha}}\right) \underset{\sim}{w}=\frac{1}{2 \lambda_{\alpha}^{2}}{ }^{t} \bar{\sim}\left(2 \lambda_{\alpha}^{2} A+\lambda_{\alpha} i B\right) \underset{\sim}{w}=\frac{1}{2 \lambda_{\alpha}^{2}}{ }^{t} \bar{\sim}\left(\lambda_{\alpha}^{2} A+C\right) \underset{\sim}{w},
$$

ensuring the positiveness of $(z, z) \forall z \in \mathcal{N}_{\alpha}$.

The prescriptions (17) —one for each subspace $\mathcal{N}_{\alpha}$ - can be glued into a single scalar product in $\mathbb{C}^{n}$, by adding the requirement of orthogonality between different subspaces.

Denoting by $\mathcal{P}_{\alpha}: \mathbb{C}^{n} \rightarrow \mathcal{N}_{\alpha}$ the family of projections associated with the direct sum decomposition (13), this leads to the expression

$$
(\underset{\sim}{z} \underset{\sim}{w})=\sum_{\alpha=1}^{r}{ }^{t}\left(\overline{\mathcal{P}_{\alpha} z}\right)\left(A+\frac{i B}{2 \lambda_{\alpha}^{2}}\right) \mathcal{P}_{\alpha} \underset{\sim}{w} \quad \forall \underset{\sim}{z} \underset{\sim}{w} \in \mathbb{C}^{n} .
$$

We then have the following.

Theorem 3. The orthonormal bases of $\mathbb{R}^{2 n}$ fulfilling the requirement (8) are in 1-1 correspondence with the orthonormal bases of $\mathbb{C}^{n}$ fulfilling the requirement (15).

Proof. Due to the orthogonal character of both direct sum decompositions $R^{2 n}=\oplus_{\alpha} \mathcal{S}_{\alpha}, \mathbb{C}^{n}=\oplus_{\alpha} \mathcal{N}_{\alpha}$, it is sufficient to discuss the relation between bases in $\mathcal{S}_{\alpha}$ and bases in $\mathcal{N}_{\alpha}$.

To this end, recalling Eqs. (9) and (10) and adapting once again the notation, we represent each pair of partner vectors of the basis $\left\{\underset{\sim}{u}(\alpha), \underset{\sim}{v}\left(i_{\alpha}\right), \alpha=1, \ldots, n_{\alpha}\right\}$ in the form

$$
\underset{\sim}{u} i_{i_{\alpha}}^{(\alpha)}=\left(\begin{array}{c}
\underset{\sim}{x} i_{i_{\alpha}}^{(\alpha)} \\
\lambda_{\alpha} y_{i_{\alpha}}^{(\alpha)}
\end{array}\right), \quad \underset{\sim}{v}(\alpha)=\left(\begin{array}{c}
v_{i_{\alpha}}^{(\alpha)} \\
\underset{i_{\alpha}}{(\alpha)} \\
-\lambda_{\alpha} \underset{\sim}{x} i_{i_{\alpha}}^{(\alpha)}
\end{array}\right) .
$$

From the Proof of Theorem 2, we know that the complex vectors $\underset{\sim}{z_{i_{\alpha}}^{(\alpha)}}=\underset{\sim}{x_{i_{\alpha}}^{(\alpha)}}+\underset{\sim}{i} \underset{i_{\alpha}}{(\alpha)}$ span $\mathcal{N}_{\alpha}$ : all we have to do is therefore to check that the correspondence $\underset{\sim}{u}(\alpha), \underset{\sim}{v}(\alpha) \rightarrow \underset{\sim}{z_{i_{\alpha}}}(\alpha)$ preserves the orthonormality relations. And, in fact,

- the definition of $\mathcal{N}_{\alpha}$ as the kernel of the operator $C-i \lambda_{\alpha} B-\lambda_{\alpha}^{2} A$ implies the identities

$$
{ }^{t} z_{i_{\alpha}}^{(\alpha)}\left(C-i \lambda_{\alpha} B-\lambda_{\alpha}^{2} A\right) \underset{\sim}{j_{\alpha}}(\alpha)=0 \quad \forall i_{\alpha}, j_{\alpha}=1, \ldots, n_{\alpha} .
$$


Due to the symmetry properties of the matrices $A, B, C$, the latter split into the pair of expressions

$$
{ }_{\sim}^{t} z_{i_{\alpha}}^{(\alpha)}\left(C-\lambda_{\alpha}^{2} A\right) \underset{\sim}{j_{\alpha}}(\alpha)=0, \quad{ }_{\sim}^{t} z_{i_{\alpha}}^{(\alpha)} B \underset{\sim j_{\alpha}}{(\alpha)}=0 .
$$

From the second one, setting $\underset{\sim}{z_{i_{\alpha}}^{(\alpha)}}=\underset{\sim}{x_{i_{\alpha}}^{(\alpha)}}+\underset{\sim}{i \underset{i_{\alpha}}{(\alpha)}}$, we get the equalities

$$
\begin{aligned}
& { }_{\sim}^{t}{\underset{\sim}{i_{\alpha}}}_{(\alpha)}^{(\alpha)}{\underset{\sim}{\sim} j_{\alpha}}_{j_{\alpha}}^{(\alpha)}={ }^{t}{ }_{\sim}^{(\alpha)} B \underset{i_{\alpha}}{y_{j_{\alpha}}^{(\alpha)}}, \\
& { }_{\sim}^{t} \underset{i_{\alpha}}{(\alpha)} B \underset{\sim}{j_{j_{\alpha}}^{(\alpha)}}+{ }^{t}{ }_{\sim}^{y_{i_{\alpha}}^{(\alpha)}} B \underset{\sim}{\underset{j_{\alpha}}{x}}(\alpha)=0 \text {. }
\end{aligned}
$$

In view of these, taking Eq. (17) into account, it is readily seen that the conditions $\left(\underset{\sim}{z} i_{\alpha}, z_{j_{\alpha}}\right)=\delta_{i_{\alpha} j_{\alpha}}$ are expressed by the equations

$$
\begin{aligned}
& { }^{t}{\underset{\sim}{i_{\alpha}}}_{i_{\alpha}} A \underset{\sim}{x_{j_{\alpha}}}+{ }^{t}{\underset{\sim}{i_{\alpha}}} A \underset{\sim}{y_{j_{\alpha}}}-\frac{1}{\lambda_{\alpha}}{ }^{t}{\underset{\sim}{i_{\alpha}}} B \underset{\sim}{y_{j_{\alpha}}}=\delta_{i_{\alpha} j_{\alpha}}, \\
& { }^{t} x_{i_{\alpha}} A \underset{\sim}{y_{j_{\alpha}}}-{ }^{t}{\underset{\sim}{i_{\alpha}}}_{i_{\alpha}} A \underset{\sim}{x_{j_{\alpha}}}+\frac{1}{\lambda_{\alpha}}{ }^{t}{ }_{\sim} i_{i_{\alpha}} B \underset{\sim}{x_{j_{\alpha}}}=0 .
\end{aligned}
$$

- Taking Eq. (8) and the properties of the operator $M$ into account, the scalar products between the basis vectors in $\mathcal{S}_{\alpha}$ read

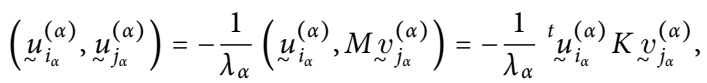

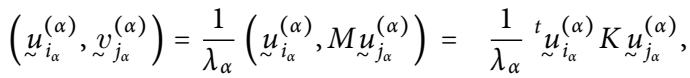

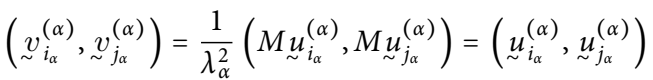

In view of Eqs. (7) and (18), the orthonormality relations therefore have the form

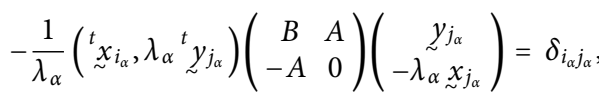

$$
\begin{aligned}
& \frac{1}{\lambda_{\alpha}}\left({ }_{\sim}^{t} x_{i_{\alpha}}, \lambda_{\alpha}{ }_{\sim}^{t} y_{j_{\alpha}}\right)\left(\begin{array}{cc}
B & A \\
-A & 0
\end{array}\right)\left(\begin{array}{c}
\underset{\sim}{x_{j_{\alpha}}} \\
\lambda_{\alpha} \\
\underset{\sim}{y_{j_{\alpha}}}
\end{array}\right)=0
\end{aligned}
$$

identical to Eqs. (19a) and (19b).

Remark 1. A deeper insight into the geometrical content of Theorem 3 is gained by considering the totality of 2-dimensional subspaces $\Sigma \subset \mathcal{S}_{\alpha}$ invariant under the action of the operator M. Each such 2-space is completely determined by the knowledge of any of its nonzero elements $\underset{\sim}{u}$, through the identification $\Sigma=\operatorname{Span}\{\underset{\sim}{\underset{\sim}{u}, M \underset{\sim}{u}\}}$.

Keeping the same notation as in Theorem 3, let us now denote by $\psi_{\alpha}: \mathcal{S}_{\alpha} \rightarrow \mathcal{N}_{\alpha}$ the correspondence sending each vector $\underset{\sim}{u}=\left(\underset{\sim}{\underset{\sim}{\lambda_{\alpha}} y}\right) \underset{\sim}{i n t o}$

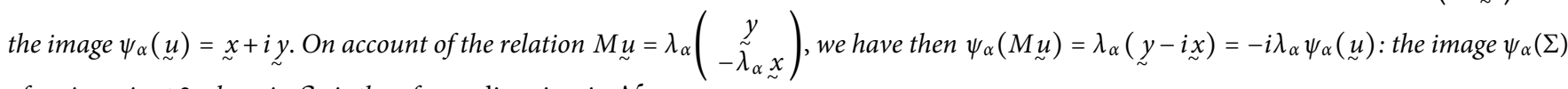
of an invariant 2-plane in $\mathcal{S}_{\alpha}$ is therefore a direction in $\mathcal{N}_{\alpha}$.

In this connection, Theorems 1 and 3 point out the following facts:

- the map $\psi_{\alpha}$ sets up a 1-1 correspondence between mutually orthogonal invariant 2-planes and mutually orthogonal directions;

- each invariant 2-plane $\Sigma \subset \mathcal{S}_{\alpha}$ admits a 1-parameter family of orthonormal bases $\underset{\sim}{\mathcal{u}}, \underset{\sim}{v}$, defined up to an arbitrary rotation

$$
\left\{\begin{array}{l}
{\underset{\sim}{u}}^{\prime}=\underset{\sim}{u} \cos \varphi-\underset{\sim}{v} \sin \varphi \\
\sim_{\sim}^{v^{\prime}}=\underset{\sim}{u} \sin \varphi+\underset{\sim}{v} \cos \varphi
\end{array}\right.
$$

The resulting direction $\psi(\Sigma) \subset \mathcal{N}_{\alpha}$ is similarly generated by a unit vector $\underset{\sim}{z}=\psi(\underset{\sim}{u})$, defined up to a phase factor $\underset{\sim}{z^{\prime}}=e^{i \varphi} \underset{\sim}{z}$.

Remark 2. The kth normal harmonic involved in the representation (12) of the solutions of the linearized equations of motion coincides with the traditional one if and only if the vectors $\underset{\sim}{h_{k}}$ and $\underset{\sim}{r_{k}}$ are parallel, i.e., if and only if the sum $\underset{\sim}{z_{k}}=\underset{\sim}{h_{k}}+\underset{\sim}{i} r_{k}$ is proportional to a real vector. In view of Eq. (11), this is possible if and only if the system 


$$
\left\{\begin{array}{l}
\left(C-\omega^{2} A\right) \underset{\sim}{z}=0, \\
B_{\sim} z=0
\end{array}\right.
$$

admits nontrivial solutions. The simplest instance occurs in the case $B=0$, corresponding to the ordinary theory of small oscillations.

\section{REFERENCES}

${ }^{1}$ E. T. Whittaker, A Treatise on the Analytical Dynamics of Particles and Rigid Bodies (University Press, Cambridge, 1917).

${ }^{2}$ T. Levi-Civita and U. Amaldi, Lezioni di Meccanica Razionale (Zanichelli, Bologna, 1984).

${ }^{3}$ B. Finzi, Meccanica Razionale (Zanichelli, Bologna, 1965), Vol. 2.

${ }^{4}$ G. Grioli, Lezioni di Meccanica Razionale (Edizioni Libreria Cortina, Padova, 1985).

${ }^{5}$ A. Fasano and S. Marmi, Analytical Mechanics: An Introduction (Oxford University Press, New York, 2006).

${ }^{6}$ F. R. Gantmacher, Lectures in Analytical Mechanics (MIR Publishers, Moscow, 1975).

${ }^{7}$ H. Goldstein, Classical Mechanics (Addison-Wesley Publishing Company, Massachusetts, USA, 1951).

${ }^{8}$ L. D. Landau and E. M. Lifshitz, Mechanics (Pergamon Press, 1969).

${ }^{9}$ D. T. Greenwood, Classical Dynamics (Dover Publications, Inc., New York, 1997).

${ }^{10}$ E. Massa and E. Pagani, Ann. Inst. Henri Poincaré 61, 17-62 (1994).

${ }^{11}$ In the rest frame of the constraints, the quadratic polynomial $L_{2}$ represents the kinetic energy of the system, the sum $U=L_{1}+L_{0}$ expresses the so-called generalized potential, and the components $Q_{k}$ collect the dynamical effects not included in $U$. Although not strictly relevant to the subsequent discussion, we recall that, in the language of analytical mechanics, denoting by $\mathcal{V}_{n+1} \stackrel{t}{\rightarrow} \mathbb{R}$ the configuration space-time of the given system, ${ }^{10}$ and identifying the first jet-bundle $j_{1}\left(\mathcal{V}_{n+1}\right)$ with the velocity spacenamely, with the affine submanifold of $T\left(\mathcal{V}_{n+1}\right)$ formed by the totality of vectors satisfying $\langle X, d t\rangle=0$-a generalized potential is essentially a 1 -form $\omega=c d t+b_{i} d q^{i}$ on $\mathcal{V}_{n+1}$, converted into a linear polynomial over $j_{1}\left(\mathcal{V}_{n+1}\right)$ through the standard algorithm $U(X):=\langle\omega, X\rangle=b_{i} \dot{q}^{i}+c$. In a similar way, the generalized forces $Q_{k}$ are the components of a contact 1-form over $j_{1}\left(\mathcal{V}_{n+1}\right)$, expressed in coordinates as $Q=Q_{k}\left(d q^{k}-\dot{q}^{k} d t\right)$. With the stated identifications, the problem of motion is reduced to the study of the integral lines of a vector field $Z$ over $j_{1}\left(\mathcal{V}_{n+1}\right)$-commonly referred to as a semi-spray-uniquely determined by the conditions

$$
Z\lrcorner d \vartheta=Q, \quad\left\langle Z, d q^{k}-\dot{q}^{k} d t\right\rangle=0, k=1, \ldots, n,
$$

$\vartheta=L d t+\frac{\partial L}{\partial \dot{q}^{k}}\left(d q^{k}-\dot{q}^{k} d t\right)$ denoting the Poincaré-Cartan 1-form associated with the Lagrangian $L$.

${ }^{12}$ In particular, the dimension of each $\mathcal{S}_{\alpha}$ is necessarily an even number, henceforth denoted by $2 n_{\alpha}$. 